\title{
Hábitos de saúde bucal em crianças internadas no Hospital da Criança do município de Chapecó, Santa Catarina, Brasil
}

\section{Oral health habits in children admitted to the Children's Hospital in the city of Chapecó, Santa Catarina, Brazil}

\author{
Ricardo Ballestreri* \\ GlaziellaWisoski Dal Santo** \\ Silvia Letícia Freddo ${ }^{* * *}$ \\ Deison Alencar Lucietto ${ }^{* * * *}$
}

\section{Resumo}

Manter a saúde bucal em pacientes pediátricos hospitalizados mostra-se de grande importância para a condição sistêmica desses, devido à mútua relação entre doenças bucais e infecções sistêmicas. Objetivo: este estudo descritivo transversal com abordagem quantitativa teve como objetivo avaliar os hábitos de saúde bucal de crianças e as percepções de saúde bucal dos cuidadores, a fim de compreender a importância da higiene bucal durante o período de internação hospitalar, diferenciando-o de outras características, como o convívio com a família e a escola. Sujeitos e método: a pesquisa teve como participantes crianças, representadas por seus cuidadores, admitidas no setor de internação do Hospital da Criança de Chapecó, SC, de maio a dezembro de 2014. Foi utilizado um questionário autoaplicável aos pais ou responsáveis, sobre variáveis socioeconômicas, de saúde bucal e saúde geral. Os dados coletados foram analisados no programa SPSS versão 19.0. Resultados: os resultados demostram que a principal causa de internação foi o diagnóstico de pneumonia $(37,6 \%)$. As mães constituíram o grupo mais frequente no acompanhamento hospitalar (78,5\%). Em torno de 30\% das crianças hospitalizadas já haviam passado por episódios de internação anterior e 59,4\% relataram que ninguém explicou o que a doença pela qual a criança estava hospitalizada causa em seu corpo. Apenas $8 \%$ foram orientados pelo cirurgião-dentista da relação da doença e a condição bucal.
Conclusão: observou-se uma baixa adesão aos cuidados de higiene bucal e grande desvalorização da saúde bucal no contexto da hospitalização.

Palavras-chave: Saúde bucal. Equipe Interdisciplinar. Cirurgião-Dentista. Crianças.

\section{Introdução}

A Odontologia, ao longo de seu processo histórico, deixou de utilizar apenas seus métodos curativistas de saúde e vem atuando cada vez mais na promoção de saúde e prevenção das doenças bucais. Assim se introduziram conceitos de trabalho em equipes multiprofissionais e interdisciplinares, abrangendo os mais diversos ambientes de cuidados com a saúde ${ }^{1}$.

Com isto, além do consultório, o cirurgião-dentista atua também em escolas, unidades básicas de saúde, hospitais e entidades. Nestes ambientes, a saúde bucal infantil e seus hábitos da infância influenciam na garantia de saúde bucal adulta.

No acompanhamento hospitalar infantil, durante conversas com os familiares, percebe-se a carência de informações principalmente sobre os cuida-

\footnotetext{
Cirurgião-Dentista, graduado na Unochapecó. Residente em Saúde da Família Residência Multiprofissional em Saúde da Família Universidade Federal de Santa Catarina UFSC.

Cirurgiã-Dentista, graduada na Unochapecó.

** Mestre em Saúde Pública na Ulbra e doutoranda em Saúde Pública na Unicamp.

**** Doutor em Saúde Pública ENSP/Fiocruz.
} 
dos com a saúde oral e sua higiene, e essa falta de informação associada ao descuido acabam por afetar a criança ${ }^{2}$.

Assim, agravos de saúde podem surgir, dentre eles, a cárie, que preocupa por ser a doença crônica de maior incidência na espécie humana, significando um relevante problema tanto econômico quanto público ${ }^{3}$.

Mudanças como alterações na dieta alimentar durante a hospitalização, introdução de medicamentos fora dos horários de higienização e desleixo dos pais ou responsáveis, associado à debilidade do paciente, podem levar a um agravo do seu quadro clínico, incluindo prejuízos na saúde bucal. Cabe aos profissionais de saúde realizar a orientação de saúde bucal, pois estes, muitas vezes, devido à grande demanda de trabalho e até mesmo pela falta de conhecimento específico na área de saúde bucal, acabam por negligenciar o cuidado.

A criança necessita de cuidados básicos, que incluem os sociais e os emocionais, do mesmo modo como se estivesse em sua casa. Na hospitalização, o fator estresse se acumula ao ambiente hospitalar e pelo próprio acometimento da doença, portanto, a criança tem de lidar com o ambiente hospitalar, que possui alto nível de ruído, perda de sono, luzes intensas, procedimentos ao acaso e imprevisíveis além de uma mudança drástica da rotina normal ${ }^{4}$.

Segundo o Estatuto da Criança e do Adolescente, Lei n. 8069 de 13 de julho de 1990, preconiza a universalização dos direitos das crianças e dos adolescentes no sentido de usufruírem do sistema de alojamento conjunto pediátrico, contando com o acompanhamento da mãe ou responsável durante os processos de permanência no hospital.

Com isto, analisar os hábitos de saúde bucal em crianças hospitalizadas se torna relevante para compreender o impacto dos hábitos de higiene bucal em crianças durante o período de internação, diferenciar características inerentes que influenciam no quadro clínico durante a internação, conhecer o nível de informação dos pais ou cuidadores sobre a higiene oral e apontar qual o acesso aos serviços odontológicos mais utilizados pelas crianças.

\section{Sujeitos e método}

Estudo descritivo transversal, com abordagem quantitativa, realizado no Hospital da Criança de Chapecó, SC, unidade pública de saúde de média complexidade, referência municipal e regional para o atendimento infantil, que oferta também serviços ambulatoriais, procedimentos cirúrgicos e internação hospitalar.

Os participantes da pesquisa foram crianças, representadas por seus cuidadores, admitidas no setor de internação médica do Hospital da Criança. Para o critério de inclusão, foi definido estar internado por mais de 24 horas e a idade superior a 28 dias de vida e inferior a 13 anos (ambos os gêneros), idade máxima permitida para internação infantil do hospital. Foram excluídos os pacientes hospitalizados em qualquer idade em leitos de isolamento. A pesquisa foi realizada de $1^{\circ}$ de maio a 31 de dezembro de 2014, totalizando 245 dias consecutivos.

O TCLE foi, previamente, esclarecido aos sujeitos da pesquisa a aplicação do questionário, conforme preconiza a CNS 466/2012, mantendo a confidencialidade do pesquisado e do responsável. Posteriormente, os questionários foram aplicados. Esta pesquisa foi submetida e aprovada pelo Comitê de Ética e Pesquisa envolvendo Seres Humanos, em 23 de outubro de 2013, pelo protocolo número 170/13 da Universidade Comunitária da Região de Chapecó (Unochapecó) e alterada pelo parecer consubstanciado de mesmo número, em de 17 de março de 2014.

O questionário autoaplicável, que forneceu dados referentes à identificação, nível socioeconômico, relação entre saúde bucal e geral e conhecimentos e atitudes sobre saúde bucal, baseadas em Cortines ${ }^{5}(2007)$, foi respondido pelos responsáveis da criança. Além disso, sob autorização, também foram obtidas informações do prontuário médico da criança.

A tabulação dos dados foi feita no programa Microsoft Excel e após transferência dos dados obtidos para o programa StatisticalPackage for the Social Sciences(SPSS), versão 19.0.

\section{Resultados}

O estudo foi realizado com uma amostra de 237 pacientes pediátricos internados no Hospital da Criança no ano de 2014. A faixa etária variou de um a 156 meses, correspondendo a uma idade média de 30,31 meses.

Conforme a Associação Brasileira de Empresas de Pesquisa (ABEP) e seus critérios de classificação econômica do Brasil do ano de 2014, tendo sido aplicados esses critérios para estudo, a classe A correspondeu a $4,22 \%$ (10), a classe B (35) a $14,77 \%$, a classe C (175) a $73,84 \%$ e a classe D a (17) $7,17 \%$, e não houve pertencentes à categoria $\mathrm{E}$.

Dentre as causas de internação, segundo o prontuário, 37,6\% dos hospitalizados apresentavam o diagnóstico de pneumonia, seguido de bronquite $(24,5 \%)$, e as demais causas ou doenças totalizavam $37,9 \%$, divididas em 25 doenças que foram citadas.

Em relação ao grau de parentesco do acompanhante, as mães constituíram o grupo mais frequente $(78,5 \%)$, quanto à variável de quem é o principal cuidador da criança no maior tempo quando fora do hospital, observou-se que $67,9 \%$ ficavam sobre o acompanhamento dos pais ou responsáveis, $17,3 \%$ em escola/creche e apenas $13,5 \%$ relataram que a criança passa a maior parte do tempo com outros. Neste estudo foi observado que $32 \%$ das crianças hospitalizadas já haviam passado por episódios de internação anterior, enquanto $68 \%$ passavam por 
seu primeiro episódio. Segundo os acompanhantes, $83,8 \%$ deles alegaram saber o motivo pelo qual a criança estava hospitalizada.

De acordo com os acompanhantes, sobre o motivo principal de hospitalização, 30,8\% relataram hospitalização devido à pneumonia, seguido de $15,6 \%$ por bronquite e outros $37,4 \%$ totalizavam as demais doenças.

Os acompanhantes foram questionados se algum profissional médico, enfermeira ou cirurgião-dentista havia explicado o que a doença pela qual a criança estava hospitalizada causava no corpo do paciente e 59,4\% afirmaram que nenhum profissional relatou nada a respeito. Em relação à orientação da doença e sua condição bucal, $8 \%$ relataram terem sido orientados por um cirurgião-dentista fora da unidade hospitalar, apenas $1,7 \%$ pelo médico e 0 restante $(90,3 \%)$ relatou que ninguém realizou nenhum esclarecimento a esse respeito.

Quando questionados se o medicamento que a criança utiliza pode alterar a saúde bucal, $34,2 \%$ responderam que sim, mas não sabem como; $4,6 \%$ relataram que sim, por conta do uso do antibiótico; 5,5\% responderam que sim pelo fato de causar danos aos dentes, mas não sabem explicar como; e 55,7\% disseram que o medicamento não altera a saúde bucal.

Sobre a questão envolvendo o comprometimento da saúde geral da criança pelo descuido com a saúde bucal 19,8\% responderam não saber, ou não informaram se pode ou não afetar, $10,2 \%$ relataram não haver relação entre saúde bucal e saúde geral, $32,5 \%$ relataram afetar muito a saúde geral e os demais, totalizando $37,5 \%$, declararam afetar pouco.

No aspecto sobre conhecimento de alguma doença que prejudique a cavidade bucal, $48,5 \%$ dos responsáveis entrevistados mostraram possuir conhecimento de alguma patologia bucal. Desses, $13,5 \%$ conhecem a cárie dental, 16,8\% afta, 9,3\% gengivite, $8,9 \%$ câncer de boca, o restante $(51,5 \%)$ não conhece nenhuma doença bucal.

Ao analisar a saúde bucal da criança, constatou-se que os acompanhantes classificam a saúde bucal dessa como a seguir: $44,3 \%$ como boa; $28,3 \%$ regular; $5,1 \%$ ótima; $7,2 \%$ ruim; $8 \%$ não sabe ou não informaram; 4,6\% ótima, explicando que isso ocorre por as crianças escovarem os dentes; $1,2 \%$ ótima, justificando pelo fato de a escovação ser supervisionada; e 1,3\% regular, afirmando que nem sempre a escovação é supervisionada.

Analisando a questão de a criança possuir boa higiene bucal em casa, $78,9 \%$ responderam que sim, das quais $17,7 \%$, identificou-se que é a própria criança quem realiza a higiene bucal; em $72,2 \%$, a criança realiza e o responsável auxilia e em apenas $10,1 \%$ dos casos são outros responsáveis ou cuidadores que auxiliam a higienização.

Dentre essas crianças, a maior parte $(62,9 \%)$ realiza a higienização utilizando escova e creme dental e 36,7\% adicionaram o uso de fio dental à higienização, o que representa um número relativamente alto em relação à idade média dos hospitalizados.
No que diz respeito à higiene bucal durante a internação hospitalar, 44,3\% da amostra afirmaram que a higiene bucal é diferente no hospital, $3 \%$ ainda justifica que é diferente por não terem levado escova dental para o hospital, 52,7\% afirmam que a escovação não é diferente da realizada em casa e $56,1 \%$ acham que essa limpeza é suficiente para deixar a boca saudável.

Ao observar a Tabela 1, mais de metade da amostra (57\% das crianças) ainda não foi ao cirurgião-dentista e $43 \%$ já passou por consulta no cirurgião-dentista. Quando questionados sobre há quanto tempo foi a última visita a esse profissional, as respostas foram: 37,1\% há menos de um ano; 5,9\% entre um e dois anos. Destaque-se que 33,3\% dos atendimentos foram realizados no serviço público; $8,4 \%$ no serviço particular e apenas $1,3 \%$ por convênios.

Sobre o motivo da consulta odontológica, constatou-se que: $35,9 \%$ relataram consulta por rotina, reparos ou manutenção; $2,5 \%$ por dor, $1,7 \%$ por cavidade nos dentes; apenas $0,8 \%$ por sangramento gengival e $2,1 \%$ por outros motivos.

Tabela 1 - Relação da criança com o serviço odontológico

\begin{tabular}{|c|c|c|}
\hline Variável & $\mathrm{N}$ & $\%$ \\
\hline \multicolumn{3}{|l|}{ A criança já visitou o dentista? } \\
\hline Sim & 102 & 43,0 \\
\hline Não & 135 & 57,0 \\
\hline \multicolumn{3}{|l|}{ Há quanto tempo? } \\
\hline Há menos de um ano & 88 & 37,1 \\
\hline Entre um e dois anos & 14 & 5,9 \\
\hline \multicolumn{3}{|l|}{ Onde foi o atendimento? } \\
\hline Nunca foi ao dentista & 135 & 57,0 \\
\hline Serviço público & 79 & 33,3 \\
\hline Serviço particular & 20 & 8,4 \\
\hline Conveniado & 3 & 1,3 \\
\hline \multicolumn{3}{|l|}{ Motivo da consulta: } \\
\hline Nunca foi ao dentista & 135 & 57,0 \\
\hline Consulta de rotina, reparos ou manutenção & 85 & 35,9 \\
\hline Cavidade nos dentes & 4 & 1,7 \\
\hline Dor & 6 & 2,5 \\
\hline Sangramento gengival & 2 & 0,8 \\
\hline Outros & 5 & 2,1 \\
\hline \multicolumn{3}{|l|}{ Informou ao dentista a doença da criança? } \\
\hline Sim & 27 & 11,4 \\
\hline Não & 210 & 88,6 \\
\hline \multicolumn{3}{|l|}{$\begin{array}{l}\text { Receberam orientações de como prevenir } \\
\text { problemas bucais? }\end{array}$} \\
\hline Sim & 121 & 51,1 \\
\hline Não & 116 & 48,9 \\
\hline \multicolumn{3}{|l|}{ Seguem as orientações? } \\
\hline Não sabe ou não informou & 88 & 37,0 \\
\hline Não & 22 & 9,3 \\
\hline Pouco & 29 & 12,1 \\
\hline Mais ou menos & 67 & 28,3 \\
\hline Muito & 31 & 13,0 \\
\hline Total & 237 & 100 \\
\hline
\end{tabular}

Fonte: elaboração dos autores. 
Ao questionar-se se a criança necessita, atualmente, de tratamento odontológico, 9,7\% relataram não saber ou não informaram, 34,6\% dos acompanhantes afirmaram que a criança não necessita no momento, $18 \%$ que a criança necessita pouco, $18,6 \%$ que necessita mais ou menos e $19 \%$ afirmam que a criança necessita muito.

Quando os pais ou responsáveis foram questionados em que medida consideravam importante a presença do cirurgião-dentista na unidade hospitalar, dos 237 participantes, 233 responderam (98,3\%) que acham importante a presença do cirurgião-dentista no hospital para prevenção e promoção de saúde bucal das crianças, quando apenas $1,7 \%$ dos participantes não consideram importante.

\section{Discussão}

A análise dos resultados desta pesquisa permitiu entender qual o nível de informação que os acompanhantes dos pacientes pediátricos possuíam sobre a saúde bucal das crianças, ressaltando a relevância da presença do cirurgião-dentista na unidade hospitalar juntamente com a equipe multidisciplinar.

Nesse estudo foi constatado que $78,5 \%$ dos acompanhantes das crianças internadas são as mães, assim como o estudo de Cristo et al. ${ }^{6}$ (2005), no qual a maioria dos acompanhantes entrevistados foi representada por mães (90\%).

A figura da mãe está inserida desde o primeiro momento da infância da criança pela dependência e referência do paciente pediátrico pela mãe. Esse elo ocorre desde a gravidez e se estende até a prática odontológica, em que a formulação dos hábitos das crianças são adquiridos pelos hábitos das mães, assim, a promoção de saúde bucal também se estabelece por meio de práticas e observações da genitora. Segundo estudos ${ }^{4,7,8}$ observou-se que sempre que havia um acompanhante com as crianças na hospitalização, esse, em geral, era a mãe. Esse fato foi confirmado também em outro estudo de Madei$\mathrm{ra}^{9}(2008)$.

Entende-se que é nesse período que o paciente pediátrico está sujeito a alterações na dieta alimentar, mudanças dos hábitos de higiene oral, com produção de saliva diminuída e, consequentemente, deficiência no processo de limpeza dos dentes, o que faz a interação do desequilíbrio entre saúde e doença importante. Assim, defende-se que deve existir o fornecimento de orientações adequadas aos acompanhantes, de modo que se evitem problemas bucais por meio de medidas simples, como manter hábitos de higiene bucal.

Neste estudo foi averiguado que o motivo principal de internação, segundo prontuário, foi a pneumonia $(37,6 \%)$. Conforme estudo de Silveira ${ }^{10}$ (2010), a higiene bucal compõe a higiene, como um todo e constitui um dos mais importantes cuidados. Uma vez que as vias respiratórias inferiores podem ser colonizadas por microrganismos da cavidade oral e por microaspiração ou aspiração das secreções da orofaringe, esta relação aponta a placa dental como um dos fatores susceptíveis para a formação da pneumonia, o que justifica a necessidade do cirurgião-dentista no hospital pelas ações de promoção da saúde.

De acordo com os resultados deste estudo, a maior parte dos entrevistados possuía como doença-base a pneumonia, com isso, sugere-se uma abordagem diferenciada na orientação de higiene bucal nesses pacientes.

É comum os pais associarem o uso frequente de medicamentos durante a infância ao precário estado de saúde bucal de seus filhos. Verificou-se, neste estudo, que $34,2 \%$ dos acompanhantes acreditam que o medicamento que a criança utiliza pode alterar a saúde bucal devido ao uso dos antibióticos e outros $4,6 \%$ acreditam que pode alterar, porém, não sabem como. Tal fato também pode ser observado no estudo de Neves et al. ${ }^{11}$ (2007), em que 70,9\% dos responsáveis associaram o uso de medicamentos pediátricos com o desenvolvimento de lesões cariosas e defeitos nas estruturas dos dentes.

Apenas 13,5\% dos entrevistados desta pesquisa citou cárie como doença que afeta a cavidade oral. No estudo de Figueira, Leite ${ }^{12}$ (2008), os entrevistados foram pais de escolares e $100 \%$ dos entrevistados apontaram como problema bucal mais conhecido a cárie e o menos conhecido a doença periodontal $(18 \%)$.

Assim, os resultados deste trabalho apontam para um desconhecimento da cárie enquanto doença bucal e precisam ser mais bem trabalhados para que sejam possíveis mudanças de comportamentos em saúde bucal.

Sobre as práticas de higiene bucal realizadas em casa, observou-se o predomínio do cuidador como o responsável para auxiliar na higiene bucal da criança $(72,2 \%)$. Rodrigues et al..$^{13}$ (2011), constataram que $75,4 \%$ das crianças hospitalizadas contavam com o auxílio do responsável para a higienização bucal durante a internação, reforçando a importância de sua conscientização para intensificar a adesão de medidas de promoção e prevenção de saúde pelo público infantil.

Ximenes et al. ${ }^{4}$ (2008), observou-se que a maior parte das crianças $(90,6 \%)$ que realizava a higienização oral utilizava a escova e o creme dental, o que, segundo McDonald e Avery ${ }^{14}$ (1995), é o método mais comum para a remoção do biofilme da cavidade bucal. Isso ocorre, pois além da ação mecânica que a escova dental exerce na superfície dental, os dentifrícios possuem a propriedade de controlar a formação de tártaro, pela adição de pirofosfatos em sua fórmula, além de proporcionar ações anticárie e dessensibilização, pela ação de fluoretos.

Neste estudo, não foi registrada grande frequência do uso do fio dental, durante o período de internação hospitalar; ao contrário do estudo de 
Rodrigues et al. ${ }^{13}$ (2011), cujo uso de fio dental em crianças se apresentou cada vez maior, por ser uma das maneiras mais eficientes de remoção do biofilme interproximal, já que, nesse espaço, a escovação é insuficiente.

Verificou-se nesta pesquisa que menos da metade das crianças internadas $(43,0 \%)$ já havia recebido algum atendimento odontológico, revelando um reduzido acesso do público infantil à assistência odontológica. Esse fato também foi observado no estudo de Rodrigues et al. ${ }^{13}$ (2011), em que 90,1\% das crianças nunca foram atendidas por um cirurgião-dentista.

Para Barros, Bertoldi ${ }^{15}$ (2002), a natureza do serviço odontológico que costumam acessar, $31,4 \%$ responderam ter recebido atendimento pelo serviço público e 68,6\% receberam em serviço particular. Já no presente estudo, $57 \%$ dos entrevistados relataram nunca ter levado a criança para um cirurgião-dentista. Dos demais, 33,3\% relataram ter levado ao serviço público, $8,4 \%$ ao serviço particular e apenas $1,3 \%$ das pessoas foram atendidas por convênio.

Em relação ao motivo da última consulta odontológica da criança, Lima et al. ${ }^{16}$ (2011), identificaram que $35 \%$ alegaram problemas periodontais e $20 \%$ cárie dentária, dos quais, no presente estudo, o motivo foi $0,8 \%$ devido a sangramento gengival e $1,7 \%$ por possuir cavidades nos dentes.

Por meio dos dados obtidos nesta investigação, $52 \%$ dos acompanhantes receberam orientações de como prevenir problemas bucais, sendo estas orientações dadas pelo cirurgião-dentista fora do hospital. Contudo, nos estudos de Ximenes et al. ${ }^{4}$ (2008), $92 \%$ dos responsáveis afirmam não ter recebido orientação sobre higiene bucal durante o período de internação, resultado semelhante ao estudo de Rodrigues et al. ${ }^{13} \mathrm{em}$ que $92,3 \%$ também afirmaram não receber orientações. Nos dois estudos, essa foi realizada por médicos, enfermeiros e técnicos de enfermagem.

Lima et al. ${ }^{16}$ (2011) conduziram um estudo sobre a importância da saúde bucal na ótica de pacientes hospitalizados e observaram que $100 \%$ dos pacientes consideraram importante a presença do cirurgião-dentista em uma unidade hospitalar. Esse resultado foi semelhante ao encontrado no presente estudo, no qual 98,3\% consideram importante a presença do cirurgião-dentista juntamente da equipe multiprofissional.

Em função de tais apontamentos, a presença do cirurgião-dentista é sugerida no ambiente hospitalar com uma tentativa de sanar muitas das dificuldades e apontamentos já discutidos. Busca-se, assim, melhorar as práticas odontológicas no ambiente hospitalar para que se possa promover saúde nos aspectos biológicos, psicológicos e sociais do paciente.

Entende-se, pois, que a realização de pesquisas sobre os cuidados bucais em ambiente hospitalar reveste-se de grande importância no contexto nacional e regional, para promover mudanças positivas em saúde bucal inclusive nos momentos de fragilidade da vida das crianças.

\section{Conclusão}

Constatou-se com este estudo que há uma baixa adesão a procedimentos de higiene bucal no ambiente hospitalar, bem como uma desvalorização da saúde bucal no contexto da criança hospitalizada. Esses fatos sugerem a necessidade de abordagem de equipes multidisciplinares em que o cirurgião-dentista esteja inserido, com o intuito de enfatizar a adoção de medidas de promoção e prevenção de saúde, tanto geral como bucal, na tentativa de auxiliar na melhoria do quadro de saúde durante a hospitalização, sendo essencial incluir no seu cuidado a criança, o cuidador e os profissionais da saúde.

O cirurgião-dentista deve ser um elo entre criança, cuidadores e demais profissionais da saúde. Quando essa se configurar numa prática institucional, haverá a ressignificação da saúde bucal como imprescindível e inseparável da saúde geral dos pacientes. Assim, espera-se fomentar um cuidado integral e humanizado do paciente hospitalizado, atuando de forma humanizada e ampliando a atuação em saúde.

\section{Abstract}

Maintaining the oral health of hospitalized pediatric patients appears to be of great importance for their systemic condition, due to the mutual relationship between oral diseases and systemic infections. Objective: this cross-sectional, descriptive, and quantitative study aimed to evaluate the oral health habits of children and the oral health perceptions of caregivers, in order to understand the importance of oral hygiene during hospital stay, differentiating it from other characteristics such as family and school life. Subjects and method: the subjects of the research were children - represented by their caregivers, admitted to the inpatient ward of the Children's Hospital of Chapecó, SC, Brazil, from May to December of 2014. A self-administered questionnaire on socioeconomic, oral health, and general health variables was provided to the parents or guardians. Data were analyzed using the SPSS software version 19.0. Results: the results show that the leading cause of hospitalization was the diagnosis of pneumonia (37.6\%). Mothers were the most frequent group at hospital monitoring (78.5\%). About $30 \%$ of hospitalized children had already been through episodes of hospitalization and 59.4\% reported that no one had explained them the consequences of the disease for which they were admitted. Only $8 \%$ received instructions from the dental surgeon regarding the relationship between the disease and the oral health condition. Conclusion: a low adherence to oral hygiene care was observed, as well as a great disregard for oral health in the context of hospitalization.

Keywords: Oral Health. Interdisciplinary Team. Dental surgeon. Children. 


\section{Referências}

1. Johnsonn J, Lethbridge J, Mullen P, Bartholomew K. The Health Care Institution as a setting for health promotion. In: Poland BD, Green LW, Rootman I. Settings for health promotion: linking theory and practice. California: Sag Publications, Inc.; 2000.

2. Potter PA, Perry AG. Fundamentos de Enfermagem. 4. ed. Rio de Janeiro: Guanabara Koogan; 1999.

3. Lorenzo JL. Microbiologia para o Estudante de Odontologia. 1. ed. São Paulo: Atheneu; 2004.

4. Ximenes RCC, Aragão DSF, Colares V. Avaliação dos cuidados com a saúde oral de crianças hospitalizadas. Rev Fac Odontol Porto Alegre 2008; 49(1):21-5.

5. Cortines AA. O. Saúde bucal em crianças hospitalizadas: percepções e atitudes de acompanhantes [Dissertação de Mestrado]. Goiânia: Faculdade de Odontologia da Universidade Federal de Goiás; 2007.

6. Cristo RC, Mello MDA, Berbet EFV, Braga TG, Kamada IK. O acompanhamento no setor pediátrico de um hospital escola: uma atividade de extensão e pesquisa. Rev Soc Bras Enferm Ped 2005; 5(2):25-34.

7. Sampaio CEP, Ventura DSO, Batista IF, Antunes TCS. Sentimento dos acompanhantes de crianças submetidas a procedimentos cirúrgicos: vivências no perioperatório. Rev Min Enferm 2009; 13(4):558-64.

8. Barbosa AM,Ribeiro DM, Caldo-Teixeira AS. Conhecimentos e práticas em saúde bucal com crianças hospitalizadas com câncer. Ciência \& Saúde Coletiva 2010; 15(1):1113-22.

9. Madeira LM. Alta Hospitalar da Criança: implicações para a Enfermagem [Dissertação de Mestrado]. São Paulo: Faculdade de Saúde Pública da Universidade de São Paulo; 1998.

10. Silveira IR, Maia FOM, Gnatta JR, Lacerda RA. Higiene bucal: prática relevante na prevenção de pneumonia hospitalar em pacientes em estado crítico. Acta Paul Enferm 2010; 23(5):697-700.

11. Neves BG, Pierro VSS, Maia LC. Percepções e atitudes de responsáveis por crianças frente ao uso de medicamentos infantis e sua relação com cárie e erosão dentária. Ciência \& Saúde Coletiva 2007; 12(5):1295-300.

12. Figueira TR, Leite ICG. Conhecimentos e práticas de pais quanto à saúde bucal e suas influências sobre os cuidados dispensados aos filhos. Pesq Bras Odontoped Clin Integr 2008; 8(1):87-92.

13. Rodrigues VP, Lopes FF, Abreu TQ, Neves MIR, Cardoso NC Avaliação dos hábitos de higiene bucal de crianças durante o período de internação hospitalar. Odontol Clín Cient 2011; 10(1):50-53.

14. McDonald R, Avery D. Odontopediatria. 6. ed. Rio de Janeiro: Guanabara Koogan; 1995.

15. Barros AJD, Bertoldi AD. Desigualdades na utilização e no acesso a serviços odontológicos: uma avaliação em nível nacional. Ciência \& Saúde Coletiva 2002; 7(4):709-17.

16. Lima DC, Saliba NA, Garbim AJI, Fernandes LAF, Garbim CAS. A importância da saúde bucal na ótica de pacientes hospitalizados. Ciência \& Saúde Coletiva 2011; 16(1):1173-80.

\section{Endereço para correspondência:}

Ricardo Ballestreri

Avenida Sete de Setembro, 633, AP 101 Centro 89874-000 Maravilha-SC

Fone: 49-9900-1980

E-mail: ricaodonto@unochapeco.edu.br

Recebido: 07/12/2015. Aceito: 28/09/2016. 